\title{
Qualidade física do solo sob diferentes sistemas de manejo e aplicação de dejeto líquido suíno
}

\author{
Dener M. S. Oliveira ${ }^{1}$, Renato P. de Lima $^{2}$ \& Ernst E. Jan Verburg ${ }^{3}$
}

\section{Palavras-chave:}

compactação do solo

resíduos agrícolas

resistência à penetração

\begin{abstract}
R E S U M O
A aplicação em solos agrícolas tem sido a principal alternativa para destinar o grande volume de dejetos líquidos suínos (DLS) gerados nos ambientes de produção. O objetivo deste trabalho foi avaliar possíveis modificações nos atributos físicos do solo decorrentes do sistema de manejo e aplicação de DLS. O estudo foi desenvolvido no município de Arapoti, PR, em agosto de 2012. Foram amostradas cinco áreas: floresta nativa, pastagem com adubação química e aplicação de DLS, plantio convencional com adubação química e aplicação de DLS, plantio direto com adubação química, plantio direto com adubação química e aplicação de DLS. Os atributos físicos do solo avaliados foram: densidade do solo (Ds), porosidade total $(\mathrm{Pt})$, macroporosidade $(\mathrm{Ma})$, razão macro e microporosidade $(\mathrm{Ma} /$ $\mathrm{Mi})$ e resistência à penetração $(\mathrm{RP})$. A adoção dos diferentes sistemas de manejo agrícola alterou a qualidade física do solo elevando a Ds e a RP e reduzindo o volume de poros. $\mathrm{O}$ solo manejado sob pastagem apresentou os maiores valores de RP. Não foram constatadas alterações nos atributos físicos do solo pela aplicação de DLS nos diferentes sistemas de manejo avaliados.
\end{abstract}

\section{Key words:}

soil compaction

agricultural wastes

penetration resistance

\section{Physical quality of soil under different systems of tillage and application of pig slurry}

\begin{abstract}
A B S T R A C T
The application in agriculture has been the main alternative to allocate the large volume of pig slurry (PS) generated in production environments. The aim of this study was to assess possible changes in physical properties of soil resulting from the management system and PS application. Five areas were sampled: native forest, pasture with mineral fertilizer and PS application, conventional tillage with mineral fertilizer and PS application, no-tillage with mineral fertilizer, no-tillage with mineral fertilizer and PS application. The evaluated physical properties of soil were: bulk density $(\mathrm{Bd})$, total porosity $(\mathrm{Pt})$, macroporosity $(\mathrm{Ma})$, macro and microporosity ratio $(\mathrm{Ma} / \mathrm{Mi})$ and penetration resistance $(\mathrm{PR})$. The adoption of different agricultural management systems altered the physical conditions of the soil, increasing the $\mathrm{Bd}$ and the $\mathrm{PR}$ and reducing the pore volume. The soil managed under pasture showed the highest values of PR. No changes were found in physical properties of soil by the application of PS in evaluated tillage systems.
\end{abstract}

Protocolo 080-2014 - 12/03/2014 • Aprovado em 31/10/2014 • Publicado em 26/01/2015

${ }^{1}$ LSN/ESALQ-USP. Piracicaba, SP. E-mail: denermsoliveira@gmail.com (Autor correspondente)

${ }^{2}$ LSN/ESALQ-USP. Piracicaba, SP. E-mail: renato_agro_@hotmail.com

${ }^{3}$ DPS/UFV. Viçosa, MG. E-mail: ernst.e.j.verburg@gmail.com 


\section{INTRODUÇÃo}

Os sucessivos problemas acerca da conservação do solo levaram os pesquisadores a desenvolver várias técnicas de manejo a fim de se evitar a degradação física do solo por processos de compactação e perdas de solo. No Brasil se encontram áreas manejadas comumente sob sistema convencional, plantio direto e cultivo mínimo (Betioli Júnior et al., 2012). Há, também, os sítios utilizados pelo setor agropecuário que mantêm grandes áreas sob o sistema de pastagem (Bilibio et al., 2010).

A utilização dos diferentes sistemas de manejo proporciona principalmente alterações na estrutura do solo (Tavares Filho \& Tessier, 2010). Em longo prazo, tal aspecto pode estar associado à formação de camadas compactadas (Lima et al., 2013). A compactação pode elevar à densidade e reduzir o volume de poros no solo, o que resultariam em aumento da erosão, perda na condutividade hidráulica e redução do alongamento radicular das plantas (Shi et al., 2012).

Comparações entre sistemas de manejo têm sido abordadas na literatura e demonstram alterações nos atributos físicos do solo ocasionados pelas diferentes formas de cultivo (Tavares Filho \& Tessier, 2010; Torres et al., 2011). Torres et al. (2011) estudaram sistemas de manejo e verificaram que os mesmos alteram, de forma diferente, os atributos físicos do solo, fato que se deve sobretudo ao revolvimento, sistemas de conservação e tráfego aplicados os quais, dependendo da intensidade, promovem alterações físicas distintas no solo ao longo do tempo.

Cunha et al. (2011) estudaram atributos físicos do solo em sistemas de preparo e culturas de cobertura na produção orgânica de feijão e milho e observaram que os sistemas de preparo do solo divergiram quanto ao seu efeito nos índices de agregação e na resistência do solo à penetração. Bilibio et al. (2010) avaliaram atributos físicos do solo em diferentes sistemas de manejo e concluíram que o uso intensivo do solo promove compactação subsuperficial indicada pela redução da relação macro/microporos, acompanhada pelo aumento da densidade do solo.

Segundo Betioli Júnior et al. (2012), uma das formas de se incrementar a qualidade estrutural do solo é por meio da conservação e adição de matéria orgânica no solo. A adição de compostos orgânicos em diferentes sistemas de manejo pode trazer não só benefícios químicos mas também melhoria na qualidade física do solo. A matéria orgânica pode reduzir a densidade do solo, elevar o volume de poros, favorecendo a infiltração e aeração para desenvolvimento das plantas (Jordan et al., 2010; Cunha et al., 2011).
Dentre os compostos orgânicos produzidos em grandes quantidades e que necessitam de um destino ambiental adequado, se destacam os dejetos líquidos suínos (DLS). A forma mais comum de utilização do DLS é a sua aplicação na agricultura, na fertilização de culturas sob plantio convencional (PC), plantio direto (PD) e pastagens (PA) (Arruda et al., 2010; Girotto et al., 2013). Além de alterações químicas, a aplicação de DLS pode estar associada a mudanças na qualidade física do solo.

Em estudo conduzido por Rauber et al. (2012), no qual os autores avaliaram propriedades físicas do solo e carbono orgânico em áreas submetidas à aplicação de dejetos suínos, foi constatado que o uso deste fertilizante orgânico promoveu melhorias na estrutura do solo, refletidas em decréscimos na densidade e resistência à penetração, aspecto também observado por Zhao et al. (2009). No entanto, os trabalhos têm mostrado que esta melhoria depende da aplicação, em longo prazo, e que outros fatores, como revolvimento do solo e tráfego agrícola, podem ser preponderantes (Zhao et al., 2009).

Avaliando a estrutura do solo em áreas submetidas a doses de DLS em semeadura direta, Arruda et al. (2010) observaram que os atributos físicos do solo e o teor de carbono orgânico não foram modificados indicando que o uso agrícola dos DLS, nas condições estudadas, mantém inalterados os atributos físicos do solo.

A literatura é rica em trabalhos que reportam aos efeitos de diferentes sistemas de manejo sobre os atributos físicos do solo, porém poucos são os estudos que avaliam atributos físicos do solo de áreas submetidas à aplicação sucessiva de DLS (Arruda et al., 2010; Costa et al., 2011).

Objetivou-se, neste trabalho, avaliar prováveis modificações em atributos físicos do solo decorrentes do sistema de manejo e da aplicação de dejeto líquido suíno.

\section{Material e Métodos}

O estudo foi desenvolvido em Arapoti, PR, em agosto de 2012, a $960 \mathrm{~m}$ de altitude, sob as coordenadas $24^{\circ} 09^{\prime} 28^{\prime \prime} \mathrm{S}$ e $49^{\circ} 49^{\prime} 37^{\prime \prime}$ O. O município está localizado na microrregião de Jaguariaíva e o clima é classificado como Cfa, segundo Köppen. A temperatura média é de $18,1^{\circ} \mathrm{C}$ com pluviosidade média anual de aproximadamente $1330 \mathrm{~mm}$ (IAPAR, 1994) .

Foram selecionadas áreas com diferentes sistemas de manejo em propriedades comerciais da região, sob Latossolo Vermelho distrófico (EMBRAPA, 2013), cujas características de manejo adotadas se encontram na Tabela 1. O solo das áreas avaliadas apresenta textura muito argilosa. A paisagem

Tabela 1. Caracterização dos sistemas de manejo adotados nas áreas experimentais, Arapoti, PR

\begin{tabular}{|c|c|}
\hline Sistemas de manejo ${ }^{(1)}$ & Descrição da área \\
\hline FL & Área de mata nativa adjacente às áreas de plantio em processo de sucessão secundária há mais de 30 anos. \\
\hline$P A+D L S$ & 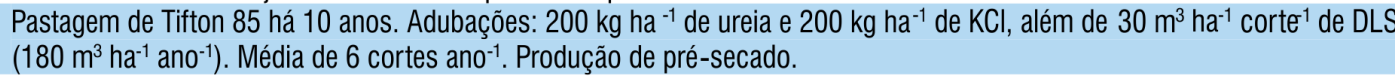 \\
\hline$P D+D L S$ & $\begin{array}{l}\text { Plantio direto há } 20 \text { anos. Sucessão soja -trigo-milho. Adubações de } 300 \mathrm{~kg} \mathrm{ha}^{-1} \text { da formulação NPK 10-20-20, acrescido de } \\
200 \mathrm{~kg}^{-1} \mathrm{~d}^{-1} \text { de ureia e } 50 \mathrm{~m}^{3} \mathrm{ha}^{-1} \text { de DLS anuais. }\end{array}$ \\
\hline PD & $\begin{array}{l}\text { Plantio direto há mais de } 30 \text { anos. Sucessão milho -aveia-soja. Adubações anuais de } 300 \mathrm{~kg} \mathrm{ha}^{-1} \text { da formulação NPK } 10-30 \\
10 \text {, mais } 100 \mathrm{~kg} \mathrm{ha}^{-1} \text { de ureia e } 100 \mathrm{~kg} \mathrm{ha}^{-1} \mathrm{de} \mathrm{KCl} \text {. }\end{array}$ \\
\hline $\mathrm{PC}+\mathrm{DLS}$ & $\begin{array}{l}\text { Plantio convencional há aproximadamente } 40 \text { anos. Sucessão milho-azevém-milho. Adubações de } 200 \mathrm{~kg} \mathrm{ha}^{-1} \text { de DAP, } 200 \\
\mathrm{~kg} \mathrm{ha}^{-1} \mathrm{KCl} \text { e } 250 \mathrm{~kg} \mathrm{ha}^{-1} \text { de ureia. Aplicação de } 120 \mathrm{~m}^{3} \mathrm{ha}^{-1} \mathrm{de} \mathrm{DLS} \text { anuais. }\end{array}$ \\
\hline
\end{tabular}

(1) FL - Floresta nativa; PA - pastagem; DLS - dejetos líquidos suínos; PD - plantio direto; PC - plantio convencional 
da região esboça características de relevo suave ondulado, porém as parcelas experimentais foram alocadas na tentativa de se preservar, ao máximo, cotas altimétricas semelhantes.

Nas áreas experimentais o DLS é aplicado a lanço e as doses baseadas em complemento à adubação mineral, principalmente do N. Além disso, os produtores vêm adequando a quantidade de DLS aplicada de acordo com as produtividades obtidas em cultivos anteriores. As doses apresentadas são uma média das quantidades aplicadas nos últimos anos. Segue uma tabela com composição química média de DLS (Tabela 2).

Cada área ou sistema de manejo foi dividida em três blocos e em cada bloco foram abertas cinco trincheiras de $0,50 \mathrm{~m}$ de profundidade. Em cada trincheira foram coletadas amostras indeformadas com aneis de volume conhecido, nas profundidades de 0-0,10,0,10-0,20 e 0,20-0,40 m, totalizando 45 amostras por sistema de manejo, em que foram determinados os seguintes atributos físicos do solo: densidade do solo $\left(\mathrm{Mg} \mathrm{m}^{-3}\right)$; macroporosidade $\left(\mathrm{m}^{3} \mathrm{~m}^{-3}\right)$; microporosidade $\left(\mathrm{m}^{3} \mathrm{~m}^{-3}\right)$ e porosidade total $\left(\mathrm{m}^{3} \mathrm{~m}^{-3}\right)$.

A microporosidade, macroporosidade e porosidade total foram determinadas pelo método da mesa de tensão, segundo EMBRAPA (1997). A porosidade total foi determinada pelo conteúdo de água na amostra saturada, a microporosidade por drenagem com a aplicação da sucção em $-6 \mathrm{kPa}$ e a macroporosidade por diferença entre a porosidade total e a microporosidade.

A densidade do solo foi determinada pelo método do anel volumétrico com base na relação massa/volume, após o procedimento, para determinação das porosidades, em que foram utilizados os mesmos aneis. As amostras foram secas em estufa a $105{ }^{\circ} \mathrm{C}$, por $24 \mathrm{~h}$. A densidade de partículas foi determinada pelo método do balão volumétrico. Todos os procedimentos foram realizados conforme EMBRAPA (1997).

Também foram realizadas amostragens para determinação da resistência à penetração (RP), com 60 medições por sistema de manejo (20 em cada uma das três glebas). Para a coleta dos dados de RP foi utilizado um penetrômetro de impacto modelo IAA/Planalsucar, com metodologia sugerida por Stolf (1991). A determinação com o penetrômetro foi realizada até uma profundidade máxima de $0,4 \mathrm{~m}$ e analisada a cada 0,05 $\mathrm{m}$ de profundidade para cada um dos sistemas de manejo. As avaliações com o penetrômetro foram realizadas em dois dias consecutivos a fim de se evitar variações na umidade do solo.

A normalidade dos dados foi testada por meio do teste Shapiro-Wilk. Os dados foram submetidos à análise de variância considerando-se um esquema experimental em blocos casualizados, com cinco tratamentos (sistemas de manejo) e três repetições (blocos). Para comparação das médias foi utilizado o teste de Tukey a 0,05 de probabilidade. Todas as

Tabela 2. Caracterização química de dejeto líquido de suíno realizada anualmente ao longo de 11 anos agrícolas

\begin{tabular}{lccccccc}
\hline & $\mathbf{p H}$ & $\mathbf{N}$ & $\mathbf{K}$ & $\mathbf{P}$ & $\mathbf{C a}$ & $\mathbf{M g}$ & $\mathbf{C O T}$ \\
\cline { 2 - 8 } & \multicolumn{7}{c}{$\mathbf{~ k g ~ m}^{-3}$} \\
Média & 7,18 & 3,56 & 1,54 & 1,39 & 1,93 & 0,76 & 22,17 \\
Desvio-padrão & 0,29 & 1,21 & 0,40 & 0,59 & 0,67 & 0,22 & 6,76 \\
\hline
\end{tabular}

COT - carbono orgânico total. Adaptado de Mafra et al. (2014) análises estatísticas foram realizadas utilizando-se o software estatístico Sisvar, versão 5.3 (Ferreira, 2010).

\section{Resultados e Discussão}

Todos os sistemas de manejo promoveram alterações na Ds, nas três profundidades analisadas, fato este corroborado pela comparação das médias dos sistemas de manejo em relação ao solo sob floresta nativa (FL), tomado como referência. Embora a Ds tenha sofrido alterações com elevação do seu valor, os sistemas de manejo, com e sem aplicações de DLS, se comportaram de forma semelhante, sem alterações significativas (Figura 1A).

Os sistemas de manejo não diferiram entre si, mas elevaram os valores de Ds em relação ao solo sob FL. Variação semelhante foi observada por Spera et al. (2009), quando analisaram atributos físicos do solo em diferentes sistemas de manejo. Esses autores verificaram diferenças entre solos sob floresta nativa e PD para Ds, com valores de 0,88 e 1,30 Mg $\mathrm{m}^{-3}$, respectivamente.

A Pt seguiu a mesma tendência da Ds e sofreu alterações significativas em todas as camadas analisadas nos sistemas de manejo com relação ao solo sob FL. Esses resultados demonstram que o volume total de poros foi alterado pela implantação dos sistemas de manejo sem distinção decorrente da aplicação de DLS, e dos sistemas sob PA, PD ou PC, em todas as profundidades analisadas (Figura 1B).

A Pt é um importante indicador da qualidade física do solo. O maior volume de poros reflete condições em que o solo sofreu pouca ou nenhuma alteração devido à pressão pelo tráfego e revolvimento. O solo sob FL reflete a condição natural em relação ao volume total de poros. Os sistemas de manejo reduziram a $\mathrm{Pt}$, evidenciando a perda de qualidade física do solo nas áreas cultivadas, semelhante ao observado por Rossetti \& Centurion (2013).

Alterações semelhantes foram constatadas por Vasconcelos et al. (2014), quando analisaram propriedades físicas do solo em diferentes sistemas de manejo. Esses autores compararam a porosidade total em solos sob mata nativa e sistemas cultivados e observaram que houve redução no volume total de poros, com valores que foram reduzidos de 0,60 a $0,38 \mathrm{~m}^{3} \mathrm{~m}^{-3}$.

A Ma só foi alterada com relação ao solo sob FL e não apresentou diferenças estatísticas entre os sistemas de manejo. Os valores de macroporosidade são importantes, por exemplo, para diagnóstico da compactação do solo, em que a principal redução de volume de poros do solo ocorre nesta fração da porosidade total (Schjonning \& Lamande, 2010).

Em sistemas sob PD e PA, nos quais o solo passa anos sem qualquer revolvimento e sob influência do tráfego, a aplicação de dejeto suíno pode ser uma alternativa para melhoria dos indicadores físicos do solo, tal como a macroporosidade (Costa et al., 2011; Comin et al., 2013).

Nessas condições, houve redução da Ma nos solos submetidos aos diferentes sistemas de manejo com relação ao solo sob FL; entretanto, também não foram observadas diferenças entres os sistemas, evidenciando que os mesmos não diferiram quanto às modificações na Ma. Uma vez que não houve diferença entre os sistemas de manejo, a aplicação de DLS também não promoveu alterações significativas na Ma (Figura 1C). 
A.

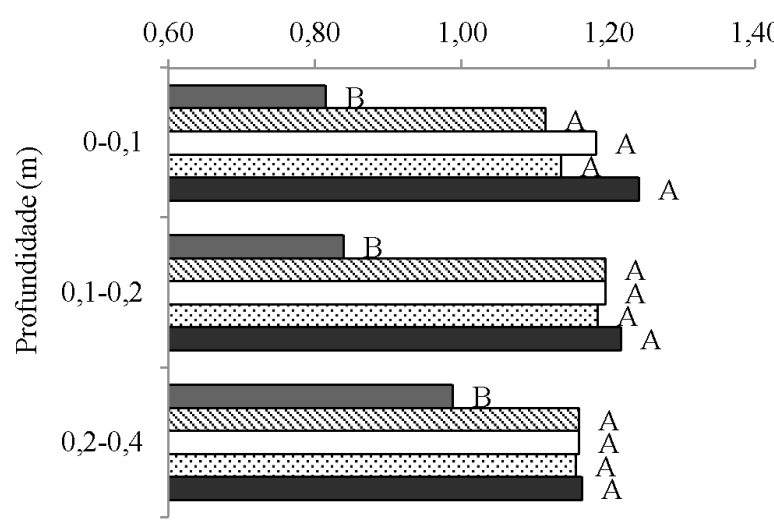

C.

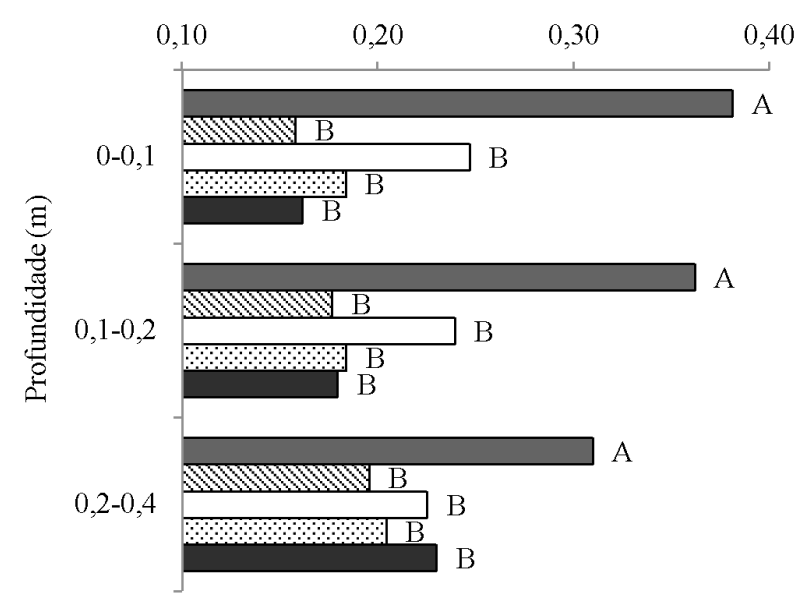

B.

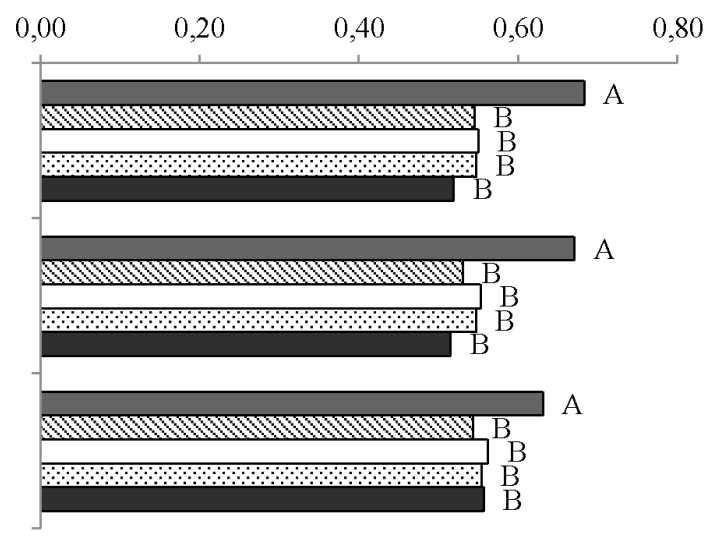

D.

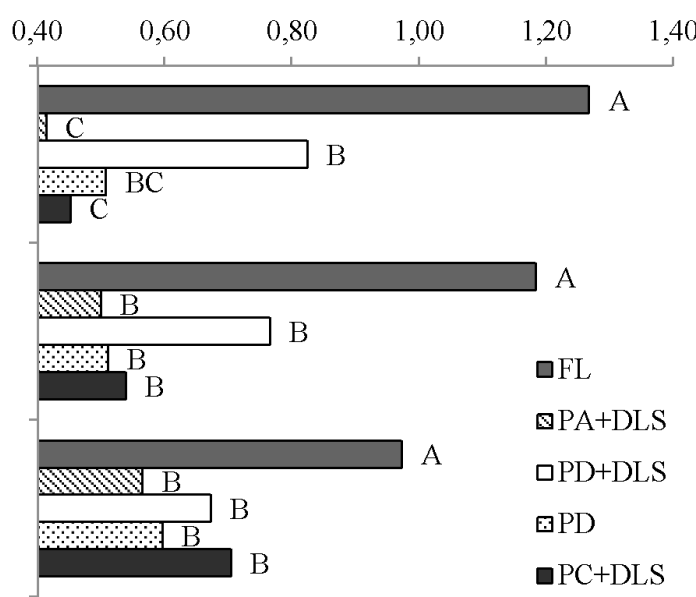

Médias seguidas de mesma letra em cada profundidade não diferem estatisticamente pelo teste de Tukey $(p<0,05)$. FL: Floresta nativa, PA: pastagem, DLS: dejetos líquidos suínos, PD: plantio direto, PC: plantio convencional

Figura 1. Densidade do solo (Ds) (A), porosidade total (Pt) (B), macroporosidade (Ma) (C) e razão macro e microporosidade $(\mathrm{Ma} / \mathrm{Mi})(\mathrm{D})$ em diferentes sistemas de manejo e aplicação de dejetos líquidos suínos

Resultados semelhantes foram observados por Costa et al. (2011), quando os autores avaliaram atributos físicos do solo e produtividade do milho sob sistemas de manejo e adubações e concluíram que o uso de DLS não apresentou potencial para melhorar as condições físicas do solo.

A adoção de sistemas de manejo favoreceu alterações na relação $\mathrm{Ma} / \mathrm{Mi}$ em todas as camadas analisadas (Figura 1D). O solo sob FL apresentou as maiores médias seguidas do PD + DLS e PD; por fim, com as menores médias e com diferenças significativas em relação às demais, o solo sob PA + DLS e PC+DLS. Esses valores sugerem que os sistemas de manejo afetaram a relação entre $\mathrm{Ma} / \mathrm{Mi}$; com alterações que dependem do sistema adotado. As demais profundidades avaliadas apresentaram diferenças entre os sistemas de manejo apenas com relação ao solo sob FL, sinalizando que essas alteração independem de qual das de manejo está sendo adotada.

A razão entre macro e microporosidade é um indicador sensível de compactação do solo; seus valores indicam o quanto de macroporos está sendo alterado em relação ao volume de microporos. Tomando como exemplo o solo sob FL, observa-se que a relação é maior que 1,0, evidenciando o maior volume de macroporos em relação ao de microporos (Figura 1D). Nos demais sistemas o volume de microporos é maior, o que pode ser uma vantagem em relação à retenção de água; no entanto, há perda de aeração e circulação de gases, aspectos que caracterizam a compactação e perda da qualidade física do solo (Rossetti \& Centurion, 2013).

Nos sistemas alterados com práticas agrícolas, o PD, com e sem aplicação de DLS, apresentou razão $\mathrm{Ma} / \mathrm{Mi}$ média acima daqueles manejados com PA e com PC. Tais resultados são proporcionados pelo menor revolvimento e aporte de resíduos orgânicos provenientes de cultivos anteriores no sistema de PD. Esta prática garante a diminuição da densidade do solo e, em contrapartida, o aumento no volume de macroporos (Betioli Júnior et al., 2012; Giarola et al., 2013), expresso muitas vezes pelo aumento na relação $\mathrm{Ma} / \mathrm{Mi}$, por se tratar de um indicador mais sensível.

O plantio convencional e o sistema sob pastagem apresentaram as menores médias da relação $\mathrm{Ma} / \mathrm{Mi}$. Esses sistemas apresentam, em comum, o fato de sofrerem constantemente pressões provenientes do tráfego. A aplicação dessas pressões é transmitida principalmente em superfície (Keller et al., 2007), aspecto passível de se associar à ausência de diferença nas camadas inferiores a $0,1 \mathrm{~m}$ (Figura 1D). Pelo tráfego, essas pressões são proveniente principalmente da pressão de insuflagem dos pneus que transmitem a pressão de calibração ao solo, fazendo com que haja redução no volume 
Tabela 3. Valores de resistência à penetração em áreas sob diferentes sistemas de manejo e aplicação de DLS

\begin{tabular}{|c|c|c|c|c|c|c|c|c|}
\hline \multirow{3}{*}{ Manejo ${ }^{(1)}$} & \multicolumn{8}{|c|}{ Profundidade (m) } \\
\hline & $0-0,05$ & $0,05-0,10$ & $0,10-0,15$ & $0,15-0,20$ & $0,20-0,25$ & $0,25-0,30$ & $0,30-0,35$ & $0,35-0,40$ \\
\hline & \multicolumn{8}{|c|}{ Resistência à penetração (MPa) } \\
\hline $\mathrm{FL}$ & $0,57 d$ & $0,85 \mathrm{C}$ & $1,31 \mathrm{~d}$ & $1,39 \mathrm{~d}$ & $1,46 \mathrm{C}$ & $1,61 \mathrm{c}$ & $1,68 b$ & $1,37 \mathrm{a}$ \\
\hline$P A+D L S$ & $2,13 \mathrm{a}$ & $3,85 \mathrm{a}$ & $4,34 \mathrm{a}$ & $4,18 \mathrm{a}$ & $3,44 \mathrm{a}$ & $3,03 \mathrm{a}$ & $2,55 \mathrm{a}$ & $1,32 \mathrm{a}$ \\
\hline$P D+D L S$ & $1,07 \mathrm{c}$ & $2,69 \mathrm{~b}$ & $3,68 b$ & $3,63 \mathrm{~b}$ & $3,08 \mathrm{~b}$ & $2,76 b$ & $2,42 \mathrm{a}$ & $1,17 \mathrm{a}$ \\
\hline PD & $1,74 b$ & $3,54 \mathrm{a}$ & $3,86 \mathrm{~b}$ & $3,56 b$ & $3,49 a$ & $3,18 \mathrm{a}$ & $2,68 \mathrm{a}$ & $1,57 \mathrm{a}$ \\
\hline$P C+D L S$ & $0,60 \mathrm{~d}$ & $1,13 \mathrm{c}$ & $2,72 \mathrm{c}$ & $2,75 \mathrm{c}$ & $3,23 a b$ & $2,81 \mathrm{~b}$ & $2,68 \mathrm{a}$ & $1,54 \mathrm{a}$ \\
\hline
\end{tabular}
pelo teste de Tukey a 0,05de probabilidade

de macroporos e aumento da microporosidade (Schjonning \& Lamande, 2010).

Os sistemas de manejo causaram elevação nos valores de $\mathrm{RP}$, sobretudo nas camadas até $0,3 \mathrm{~m}$ (Tabela 3 ). Nas camadas mais profundas o aumento com relação ao solo sobre FL é menos intenso principalmente a partir de $0,3 \mathrm{~m}$. Os maiores valores de RP foram observados para o tratamentos PA + DLS.

Comparando-se os tratamentos sob plantio direto, observase que até $0,10 \mathrm{~m}$ o tratamento $\mathrm{PD}$ apresentou média de RP maior que o PD + DLS. O PC+DLS, até a camada de 0,20 $\mathrm{m}$, apresentou os menores valores dentre os tratamentos analisados, exceto quando comparado com a FL.

Embora não haja um consenso sobre os valores de RP limitantes ao desenvolvimento das plantas, normalmente se adotam de 2,0 a 2,5 MPa (Betioli Júnior et al., 2012) como sendo valores que podem causar impedimento ao desenvolvimento radicular. Nas camadas de 0,10-0,15 m e 0,15-0,10 m, com exceção do PD + DLS e do FL, os valores de RP ficaram entre 3,0 e 4,0 MPa (Tabela 3). Tais valores são considerados altos e acima do valor limitante abordado na literatura (Vaz et al., 2011; Betioli Júnior et al., 2012; Guimarães et al., 2013).

O sistema sob pastagem apresentou os maiores valores de RP dentre todos os sistemas de manejo analisados. Esses altos valores de RP foram observados principalmente na camada de $0,1-0,2 \mathrm{~m}$, concordando com os resultados obtidos por Ralisch et al. (2008), que avaliaram a resistência à penetração de um Latossolo Vermelho Amarelo do Cerrado sob diferentes sistemas de manejo. Apesar da ausência de pisoteio de animais, o trânsito constante para corte da forragem e o não revolvimento, se associam ao resultado observado.

Observou-se, de acordo os resultados apresentados na Tabela 3, influência da aplicação de DLS na RP dos tratamentos sob plantio direto na camada de até $0,10 \mathrm{~m}$. Porém, com base nos resultados obtidos para outros atributos físicos do solo (Ds, $\mathrm{Pt}$, razão Ma/Mi e Ma), não houve mais evidencias conclusivas de que o DLS causou alterações físicas do solo, corroborando com os resultados obtidos por Costa et al. (2011) e Arruda et al. (2010).

Embora a RP seja uma importante variável indicadora da qualidade física do solo, das variáveis medidas neste estudo é a que mais pode sofrer variações da própria matriz do solo, principalmente da umidade no momento da amostragem, bem como do masuseio do penetrômetro pelo operador.

As alterações impostas por processos mecânicos, de pressão e revolvimento, de acordo com os resultados, parecem ter prevalecido sobre os efeitos da aplicação de DLS. Conclusões semelhantes foram obtidas por Costa et al. (2011) e Arruda et al. (2010) que, estudando alterações físicas após a aplicação de dejetos suínos em diferentes sistemas de manejo, concluíram que não houve influência da aplicação de DLS nos atributos físicos do solo.

Zhao et al. (2009) avaliaram atributos físicos do solo em diferentes sistemas de manejo e encontraram diferenças significativas e melhoria na qualidade física do solo após aplicação de DLS, durante 25 anos. Enfim, esses autores afirmam que alterações nas propriedades físicas do solo via aplicação de dejetos animais estão fortemente associadas ao manejo das áreas em longo prazo e ainda reforçam a ideia de que a principal contribuição da aplicação de dejetos animais para qualidade física do solo é proveniente do aumento da matéria orgânica, que eleva a quantidade de poros e diminui a densidade do solo, refletindo em melhorias na sua estrutura.

\section{Conclusões}

1. A adoção dos diferentes sistemas de manejo agrícola alterou as condições físicas do solo, elevando a densidade e a resistência à penetração e reduzindo o volume de poros.

2. O solo manejado sob pastagem apresentou os maiores valores de resistência à penetração.

3. Não foram constatadas alterações nos atributos físicos do solo decorrentes da aplicação de dejetos líquidos suínos nos diferentes sistemas de manejo avaliados.

\section{Agradecimentos}

Os autores agradecem aos Srs. Gerrit Verburg, Hendrix Verburg, Lucas Salamons e Wout Verbutg, por permitirem a amostragem em suas propriedades para a condução do presente estudo.

\section{Literatura Citada}

Arruda, C. A. O.; Alves, M. V.; Mafra, P. C. C.; Albuquerque, J. A.; Santos, J. C. P. Aplicação de dejeto suíno e estrutura de um Latossolo Vermelho sob semeadura direta. Ciência \& Agrotecnologia, v.34, p.804-809, 2010. http://dx.doi.org/10.1590/ S1413-70542010000400002

Betioli Júnior, E.; Moreira, W. H.; Tormena, C. A.; Ferreira, C. J. B.; Silva, A. P.; Giarola, N. F. B. Intervalo hídrico ótimo e grau de compactação de um Latossolo Vermelho após 30 anos sob plantio direto. Revista Brasileira de Ciência do Solo, v.36, p.971-982, 2012. http://dx.doi.org/10.1590/S0100-06832012000300027

Bilibio, W. D.; Corrêa, G. F.; Borges, E. N. Atributos físicos e químicos de um Latossolo, sob diferentes sistemas de cultivo. Ciência \& Agrotecnologia, v.34, p.817-822, 2010. http://dx.doi.org/10.1590/ S1413-70542010000400004 
Comin, J. J.; Loss, A.; Veiga, M. da; Guardini, R.; Schmitt, E.; Oliveira, P. A. V. de; Belli Filho, P.; Couto, R. da R.; Benedet, L.; Muller Júnior, V.; Brunetto, G. Physical properties and organic carbon content of a Typic Hapludult soil fertilised with pig slurry and pig litter in a no-tillage system. Soil Research, v.51, p.459-470, 2013. http://dx.doi.org/10.1071/SR13130

Costa, M. S. S. M.; Pivetta, L. A.; Costa, L. A. M.; Pivetta, L. G.; Castoldi, G.; Steiner, F. Atributos físicos do solo e produtividade do milho sob sistemas de manejo e adubações. Revista Brasileira de Engenharia Agrícola e Ambiental, v.15, p.810-815, 2011. http:// dx.doi.org/10.1590/S1415-43662011000800007

Cunha, E. Q.; Stone, L. F.; Moreira, J. A. A.; Ferreira, E. P. B.; Didonet, A. D.; Leandro, W. M. Sistemas de preparo do solo e culturas de cobertura na produção orgânica de feijão e milho. I - Atributos físicos do solo. Revista Brasileira de Ciência do Solo, v.35, p.589602, 2011. http://dx.doi.org/10.1590/S0100-06832011000200028

EMBRAPA - Empresa Brasileira de Pesquisa Agropecuária. Manual de métodos de análise de solo. Rio de Janeiro: EMBRAPA, 1997. 212p.

EMBRAPA - Empresa Brasileira de Pesquisa Agropecuária. Solos. Sistema brasileiro de classificação de solos. Rio de Janeiro: EMBRAPA, 2013. 353p.

Ferreira, D. F. Sisvar - Sistema de análise de variância. Versão 5.3. Lavras: UFLA, 2010.

Giarola, N. F. B.; Silva, A. P.; Tormena, C. A.; Guimarães, R. M. L.; Ball, B. C. On the visual evaluation of soil structure: The Brazilian experience in Oxisols under no-tillage. Soil \& Tillage Research, v.127, p.60-64, 2013. http://dx.doi.org/10.1016/j.still.2012.03.004

Girotto, E.; Ceretta, C. A.; Lourenzi, C. R.; Lorensini, F.; Tiecher, T. L.; Vieira, R. C. B.; Trentin, G.; Basso, C. J.; Miotto, A.; Brunetto, G. Nutrient transfers by leaching in a no-tillage system through soil treated with repeated pig slurry applications. Nutrient Cycling in Agroecosystems, v.95, p.115-131, 2013. http://dx.doi.org/10.1007/ s10705-013-9552-2

Guimarães, R. M. L.; Blainski, E.; Fidalski, J. Intervalo hídrico ótimo para avaliação da degradação física do solo. Revista Brasileira de Ciência do Solo, v.37, p.1512-1521, 2013. http://dx.doi. org/10.1590/S0100-06832013000600008

IAPAR - Instituto Agronômico do Estado do Paraná. Cartas climáticas do Estado do Paraná. Londrina: IAPAR, 1994. CD Rom.

Jordan, A.; Zavala, L. M.; Gil, J. Effects of mulching on soil physical properties and runoff under semi-arid conditions in southern Spain. Catena, v.81, p.77-85, 2010. http://dx.doi.org/10.1016/j. catena.2010.01.007

Keller, T.; Defossez, P.; Weisskopf, P.; Arvidsson, J.; Richard, G. SoilFlex: A model for prediction of soil stresses and soil compaction due to agricultural field traffic including a synthesis of analytical approaches. Soil \& Tillage Research, v.93, p.391-411, 2007. http://dx.doi.org/10.1016/j.still.2006.05.012

Lima, R. P.; Leon, M. J.; Silva, A. R. Compactação do solo de diferentes classes texturais em áreas de produção de cana-de-açúcar. Revista Ceres, v.60, p.16-20, 2013. http://dx.doi.org/10.1590/S0034737X2013000100003

Mafra, M. S. H.; Cassol, P. C.; Albuquerque, J. A.; Correa, J. C.; Grohskopf, M. A.; Panisson, J. Acúmulo de carbono em Latossolo adubado com dejeto líquido de suínos e cultivado em plantio direto. Pesquisa Agropecuaria Brasileira, v.49, p.630-638, 2014. http://dx.doi.org/10.1590/S0100-204X2014000800007
Ralisch, R.; Miranda, T. M.; Okumura, R. S.; Barbosa, G. M. C.; Guimarães, M. F.; Scopel, E.; Balbino, L. C. Resistência à penetração de um Latossolo Vermelho Amarelo do Cerrado sob diferentes sistemas de manejo. Revista Brasileira de Engenharia Agrícola e Ambiental, v.12, p.381-384, 2008. http://dx.doi. org/10.1590/S1415-43662008000400008

Rauber, L. P.; Piccolla, C. D.; Andrade, A. P.; Friederichs, A.; Mafra, A. L.; Corrêa, J. C.; Albuquerque, J. A. Physical properties and organic carbon content of a Rhodic Kandiudox fertilized with pig slurry and poultry litter. Revista Brasileira de Ciência do Solo, v.36, p.1323-1332, 2012. http://dx.doi.org/10.1590/S010006832012000400026

Rossetti, K. V.; Centurion, J. F. Sistemas de manejo e atributos físicohídricos de um Latossolo Vermelho cultivado com milho. Revista Brasileira de Engenharia Agrícola e Ambiental, v.17, p.472-479, 2013. http://dx.doi.org/10.1590/S1415-43662013000500002

Schjonning, P.; Lamande, M. A note on the vertical stresses near the soil-tyre interface. Soil \& Tillage Research, v.108, p.77-82, 2010. http://dx.doi.org/10.1016/j.still.2010.03.006

Shi, X. H.; Yang, X. M.; Drury, C. F.; Reynolds, W. D.; McLaughlin, N. B.; Zhang, X. P. Impact of ridge tillage on soil organic carbon and selected physical properties of a clay loam in southwestern Ontario. Soil \& Tillage Research, v.120, p.1-7, 2012. http://dx.doi. org/10.1016/j.still.2012.01.003

Spera, S. T.; Santos, H. P.; Fontaneli, R. S.; Tomm, G. O. Integração lavoura e pecuária e os atributos físicos de solo manejado sob sistema plantio direto. Revista Brasileira de Ciência do Solo, v.33, p.129-136, 2009. http://dx.doi.org/10.1590/S010006832009000100014

Stolf, R. Teoria e teste experimental de fórmulas de transformação dos dados de penetrômetro de impacto em resistência do solo. Revista Brasileira de Ciência do Solo, v.15, p.229-235, 1991.

Tavares Filho, J.; Tessier, D. Effects of different management systems on porosity of oxisols in Paraná, Brazil. Revista Brasileira de Ciência do Solo, v.34, p.899-906, 2010. http://dx.doi.org/10.1590/ S0100-06832010000300031

Torres, J. L. R.; Fabian, M. G.; Pereira, M. G. Alterações dos atributos físicos de um Latossolo Vermelho submetido a diferentes sistemas de manejo. Ciência \& Agrotecnologia, v.35, p.437-445, 2011. http://dx.doi.org/10.1590/S1413-70542011000300001

Vasconcelos, R. F. B.; Souza, E. R.; Cantalice, J. R.; Silva, L. S. Qualidade física de Latossolo Amarelo de tabuleiros costeiros em diferentes sistemas de manejo da cana-de-açúcar. Revista Brasileira de Engenharia Agrícola e Ambiental, v.18, p.381-386, 2014. http:// dx.doi.org/10.1590/S1415-43662014000400004

Vaz, C. M. P.; Manieri, J. M.; Maria, I. C.; Tuller, M. Modeling and correction of soil penetration resistance for varying soil water content. Geoderma, v.166, p.92-101, 2011. http://dx.doi. org/10.1016/j.geoderma.2011.07.016

Zhao, Y.; Wang P.; Li, J.; Chene, Y.; Yingf, X.; Liu, S. The effects of two organic manures on soil properties and crop yields on a temperate calcareous soil under a wheat-maize cropping system. European Journal of Agronomy, v.31, p.36-42, 2009. http://dx.doi. org/10.1016/j.eja.2009.03.001 